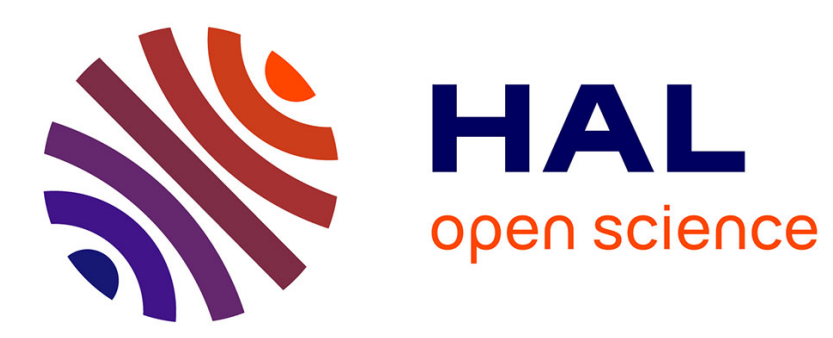

\title{
A NEXAFS Study on the Coordination Geometry of Aluminum in Minerals and Y Zeolite. Cornparison of Experiment and Theory
}

J. van Bokhoven, H. Sambe, D. Koningsberger, D. Ramaker

\section{- To cite this version:}

J. van Bokhoven, H. Sambe, D. Koningsberger, D. Ramaker. A NEXAFS Study on the Coordination Geometry of Aluminum in Minerals and Y Zeolite. Cornparison of Experiment and Theory. Journal de Physique IV Proceedings, 1997, 7 (C2), pp.C2-835-C2-840. 10.1051/jp4:1997254 . jpa-00255333

\section{HAL Id: jpa-00255333 https://hal.science/jpa-00255333}

Submitted on 1 Jan 1997

HAL is a multi-disciplinary open access archive for the deposit and dissemination of scientific research documents, whether they are published or not. The documents may come from teaching and research institutions in France or abroad, or from public or private research centers.
L'archive ouverte pluridisciplinaire HAL, est destinée au dépôt et à la diffusion de documents scientifiques de niveau recherche, publiés ou non, émanant des établissements d'enseignement et de recherche français ou étrangers, des laboratoires publics ou privés. 


\title{
A NEXAFS Study on the Coordination Geometry of Aluminum in Minerals and Y Zeolite. Comparison of Experiment and Theory
}

\author{
J.A. van Bokhoven*, H. Sambe, D.C. Koningsberger* and D.E. Ramaker
}

\author{
Chemistry Department, George Washington University, Washington, DC 20052, U.S.A. \\ * Laboratory of Inorganic Chemistry and Catalysis, Debye Institute, Utrecht University, \\ 3508 TB Utrecht, The Netherlands
}

\begin{abstract}
Curved-wave multiple scattering cluster calculations using the FEFF6 code were successfully used to interpret experimental Al K edge NEXAFS spectra of various minerals and $\mathrm{Y}$ zeolites, starting about $5 \mathrm{eV}$ above threshold. The Al octahedral, tetrahedral and square planar geometries can be easily distinguished from each other utilizing the NEXAFS data. Semiempirical molecular orbital calculations were used to interpret the whitelines below $5 \mathrm{eV}$ in the data. A new assignment is given to the NEXAFS peaks found for Al atoms having a distorted octahedral coordination. Use of NEXAFS for determining the geometrical conformations of a $\mathrm{Y}$ zeolite involving $\mathrm{Al}$ atoms with mixed conformational geometry is emphasized. In $\mathrm{H}^{+} \mathrm{Y}$ zeolite, separate contributions to the NEXAFS spectra from tetrahedrally and octahedrally coordinated Al atoms are identified. There are also strong indications for the presence of penta-coordinated $\mathrm{Al}$ atoms accompanying the non-framework octahedrally coordinated Al atoms in the $\mathrm{H}^{+} \mathrm{Y}$ zeolite. We have found that detailed interpretations of the NEXAFS data complement the EXAFS and NMR results by providing unique geometrical information on the $\mathrm{Al}$ coordination.
\end{abstract}

\section{INTRODUCTION}

Zeolites are widely used as catalysts and as catalyst supports in industrial processes. Applications can be found in processes like fluid catalytic cracking (FCC), hydrocracking, paraffin isomerization, aromatic alkylation, ion exchange resins, molecular sieves, sorbents etc.. Zeolites are open 3-dimensional framework alumino-silicates and can be represented as being derived from $\mathrm{SiO}_{2}$ by replacement of part of the $\mathrm{SiO}_{4}{ }^{-4}$ tetrahedra with $\mathrm{AlO}_{4}{ }^{-5}$ tetrahedra. Charge balance requires the presence of cations to neutralise the extra negative charge on each aluminum oxide tetrahedron. These cations are not part of the framework and can easily be exchanged. It is well-known that the aluminum tetrahedral site in acidic zeolites and the associated hydroxyl protons play a prominent role in many catalytic reactions, the latter as a Brönsted acid. The strength of this Brönsted acid is affected by several factors, like the presence of alkali and other cations, the silicon to aluminum ratio, the presence of non-framework aluminum and the structure of the zeolite ${ }^{1,2}$.

Upon dealumination of zeolite, non-framework octahedrally coordinated aluminum atoms are formed. These nonframework aluminum atoms can be distinguished from tetrahedrally coordinated (framework) aluminum by conventional $\mathrm{Al}^{27}$ NMR data (shifts of $0 \mathrm{ppm}$ and $55-65 \mathrm{ppm}$ for octahedrally and tetrahedrally coordinated aluminum respectively) ${ }^{3}$. The total amount of aluminum at all sites can be determined chemically and the aluminum distribution among the framework and nonframework aluminum sites can be ascertained from $\mathrm{Si}^{29} \mathrm{NMR}$ data. However, the distribution determined in this way is in poor agreement with the intensities of lines observed in conventional $A l^{27}$ NMR spectra. Recent $A l^{27}$ MAS NMR data gives more detail, showing the presence of additional aluminum, visible at a shift of around $30 \mathrm{ppm}$. However, the nature of this contribution has not yet been established unambiguously. It has been attributed to distorted tetrahedrally-coordinated nonframework-aluminum ${ }^{4,5}$, penta-coordinated non-framework aluminum ${ }^{6,7,9}$ and polymeric aluminum structures ${ }^{10}$. In addition, the line at around $60 \mathrm{ppm}$ has sometimes been reported to be a superposition of two signals ${ }^{9}$. The penta-coordinated Al site has been suggested to have a trigonal bipyramidal geometry ${ }^{11}$, but a square planar geometry has also been proposed .

It has been suggested that the extra non-framework (four coordinated and/or penta coordinated) Al sites may play a role in the catalytic activity since they have potential as Lewis acid centers ${ }^{7}$ or they may polarize the acidic site, enhancing acidity ${ }^{12,13}$. Measurements on steamed $Y$ and LZ-210 zeolites show catalytic activity by both framework and non-framework aluminum sites ${ }^{10}$. It is reported that in some cases, $\mathrm{Y}$ zeolite, in which framework aluminum is completely absent, still shows catalytic activity ${ }^{14}$. Clearly the determination of the local geometry, coordination number, and relative importance of the framework and non-framework nature of the active aluminum sites in zeolites is important. However, much further work needs to be done before this task is completed.

In an effort to determine the local structure of the aluminum atom in Y zeolite, previously published NEXAFS data with different cations, $\mathrm{H}^{+}, \mathrm{NH}_{4}^{+}$and $\mathrm{Na}^{+}$are interpreted in this work. Previous analysis of the EXAFS for these samples indicated that these three materials have three similar aluminum oxide bond lengths and possess primarily 4 -fold (i.e., tetrahedrally) coordinated Al sites ${ }^{13}$.

In this paper we demonstrate the usefulness of NEXAFS data for the determination of the local aluminum environment in minerals and $\mathrm{Y}$ zeolites. We perform curved-wave multiple-scattering calculations using the FEFF6 code and semi-empirical LCAO-MO calculations (i.e. the AM1 approximation using the "HyperChem" code) ${ }^{15}$ on various clusters representing the mineral or zeolite. Aluminum oxide clusters with different coordinations will be considered and the 
calculated results are compared to experimental Al K-edge NEXAFS data from a wide array of aluminum oxide minerals. From these comparisons, we recognize characteristic features in the spectra arising from the different coordinations. The coordinations we encounter are the tetrahedral and the octahedral coordinations in addition to square planar aluminum oxide.

Using the characteristic features we observe from the well characterized minerals, we will analyze the NEXAFS range of the $\mathrm{Y}$ zeolite absorption spectra mentioned above. In this analysis, we will use the NMR results, which indicate the presence of octahedral aluminum oxide in the $\mathrm{H}^{+} \mathrm{Y}$ zeolite sample. A technique to separate the different contributions in a NEXAFS spectrum having several distinct coordinations of aluminum oxides is described.

\section{CALCULATIONAL METHODS}

Curved-wave, multiple-scattering (CW-MS) cluster calculations utilizing the FEFF6 code developed by Rehr and Albers ${ }^{16,17}$ were performed in this work. For the furst row transition metals and lighter elements, the absolute energy must be shifted by $0-5 \mathrm{eV}$ for optimum agreement with experiment. Here, the FEFF6 results were optimally aligned with the experimental data.

The input parameters for the theoretical calculations include: the atomic number of each unique atom in the cluster, the coordinates of each atom in the cluster, the choice of exchange potential (Hedin-Lundquest, Dirac-Hara, or ground state), the maximum path length, criteria for the path filter which determines the amount of multiple scattering, the Debye Waller factor and the charge on the ions (only positive integers are allowed by the code, so we used zero in all cases). We employed the ground state exchange correlated potential, which does not allow for inelastic energy losses. We used a zero imaginary part in the potential, thus ignoring any lifetime or experimental broadening effects. The maximum path length was set to 8-10 $\AA$ for the 1 and 2 shell calculations, and to $15 \AA$ for the longer shell calculations. The path filter was chosen to be $2 \%$ for the plane waves and $4 \%$ for the curved waves (i.e. the default values) to include all important multiple scattering paths. The Debye Waller factor was set to be zero.

To better model the highly localized peaks very near or below the edge, which the FEFF6 code is known to underestimate, we performed LCAO-MO calculations utilizing the AMl semi-empirical hamiltonian, with a standard parametrization as provided in the Hyperchem package ${ }^{15}$. Inputs for this code are the geometrical coordinates and the electronic charge on the cluster. In order to account for the core-hole in the final state of the X-ray absorption transition, which is reflected in the NEXAFS data, the $\mathrm{Z}+1$ rule $^{18}$ was utilized; i.e. in the cluster calculations the absorbing atom was assumed to be a $\mathrm{Si}$ atom. The relative NEXAFS intensities are assumed to be equal to the relative atomic populations on this $\mathrm{Si}$ atom. The absolute populations are normalized for best agreement with the experimental data.

\section{RESULTS AND DISCUSSION}

\subsection{Standard spectra of tetrahedral and octahedral coordinations}

Experimental A1 K-edge NEXAFS data for aluminum oxide compounds with an octahedral coordination, distorted and non-distorted, are given in Figure 1. These spectra are characteristic for compounds with these structures ${ }^{19}$. The compound $\left(\mathrm{NdAlO}_{3}\right)$ which exhibits the Perovskite structure has a non-distorted octahedral Al coordination. FEFF6 calculations on an $\mathrm{AlO}_{6}$ cluster representing the Perovskite structure and on $\mathrm{SiAl}_{14} \mathrm{O}_{18}{ }^{-10}$ cluster, representing corundum are also given. Similarities between the FEFF6 results and the experimental spectra can be seen in the relative peak energies and intensities of the whiteline and the peak around $45-50 \mathrm{eV}$. The corundum structure has a distorted octahedral AI site with two different Al-O bond lengths, namely 1.86 and $1.97 \AA$ respectively ${ }^{20}$ but only one peak is visible in the $45-50 \mathrm{eV}$ region in the theory. However, the whiteline in the theory is slightly broadened and asymmetric compared to the calculated and experimental spectra for the Perovskite structures. The set of small features around $15-35 \mathrm{eV}$ in the experimental spectra are reproduced in the calculated spectrum by two broader peaks. AM1 calculations for a $\mathrm{SiO}_{6}^{-8}$ indicates that the whiteline transition in the Al NEXAFS data for structures exhibiting the Perovskite structure is a $1 a_{\mathrm{g}} \rightarrow \mathrm{t}_{\mathrm{lu}}$ transition. The $t_{\mathrm{lu}}$ final state possesses a significant $\mathrm{p}$ density of states on the silicon atom.

As established in the literature and seen in the figure, materials with distorted octahedral Al sites have edge features at 1568 and $1572 \mathrm{eV}$, while the Perovskite structure, with undistorted octahedral Al sites, has a single white line at $1570 \mathrm{eV}$ (the average of these two energies). Although the magnitude of the whiteline splitting in the distorted structures remains constant around $4 \mathrm{eV}$, the relative intensities of the two peaks vary. In addition to the split two-peak whiteline, a weak pre edge feature (at circa $-2 \mathrm{eV}$ ) can be seen in all of the spectra for Topaz ${ }^{21}$, Kyanite ${ }^{21}$, Jadeite ${ }^{21,22}$. The AM1 results for the $\mathrm{SiO}_{6}{ }^{-8}$ cluster in a corundum-like distorted conformation reveal the split whiteline and the presence of a little pre-edge feature as seen in Figs 1 and 2. The relative intensities of the peaks are in reasonable agreement with experiment, however, the magnitude of the splitting falls short in comparison with experiment. Other extended Huckel theory (EHT) calculations (with no charge iteration) on a cluster with and without d-orbitals (not shown) show an increase in splitting upon including d-orbitals on the absorbing atom. Although the amount of splitting still falls short in comparison with the experiment, this shows the importance of $\mathrm{d}$ orbitals on the splitting of the white line in distorted octahedral compounds. Based on our AM1 calculations, the 
whiteline transitions in the $\mathrm{K}$ edge Al NEXAFS data for materials with distorted octahedral Al sites can be assigned to la $\rightarrow$ $e_{u}$ and $1 a_{g} \rightarrow a_{u}$ transitions. This is in contrast to previous reports, which suggested two $1 a_{g} \rightarrow t_{u}$ transitions ${ }^{23}$, or attributed the second peak to multiple scattering contributions ${ }^{24}$ clearly not visible in the FEFF6 results.

Experimental Al K edge NEXAFS spectra for structures which have tetrahedrally coordinated aluminum sites are given in Figure 2. $\mathrm{Na}^{+} \mathrm{Y}$ zeolite and $\mathrm{NH}_{4}^{+} \mathrm{Y}$ zeolite are respectively $100 \%$ and $98 \%$ tetrahedrally coordinated according to $\mathrm{Al}^{27} \mathrm{NMR}$. Albite ${ }^{25}$ is often used as a reference spectrum for compounds with tetrahedrally coordinated aluminum oxide. It has been established that tetrahedral compounds exhibit one strong edge feature at $1566 \mathrm{eV}$ (see also ${ }^{21,22}$ ). This is $2 \mathrm{eV}$ less in comparison with the first feature in the distorted octahedral compounds ${ }^{24}$. The FEFF6 spectra for an $\mathrm{AlO}_{4}$ cluster and the AM1 results for a $\mathrm{SiO}_{4}^{-4}$ cluster are also shown. For both calculations the clusters are in a regular tetrahedral conformation with an aluminum oxygen bond length of $1.70 \AA$.

The FEFF6 results reproduce the relative peak positions and intensities of the peaks around $20 \mathrm{eV}$ and $70 \mathrm{eV}$ (not shown in the Figure) in the experimental spectra. A small peak around $45 \mathrm{eV}$ in the experimental spectra appears around 40 $\mathrm{eV}$ in the FEFF6 spectrum. The two peaks visible in the three experimental spectra around $5-10 \mathrm{eV}$ are reproduced in the FEFF 6 calculated spectrum by a single peak around $8 \mathrm{eV}$. It is likely that adding more shells in the FEFF 6 calculation would result in a spectrum in which this peak would be split into two distinct peaks. However, a clear difference between the experimental and FEFF6 spectra is the absence of the sharp whiteline in the FEFF6 result. It is known that the FEFF6 code often does not reproduce localized or bound states ${ }^{16,17}$ and therefore apparently the FEFF6 code does not reproduce this sharp whiteline for tetrahedrally coordinated aluminum oxide. The AM1 calculation on the tetrahedrally coordinated $\mathrm{SiO}_{4}{ }^{-4}$ cluster, however, indicates a single transition, $1 a_{g} \rightarrow t_{2}$, for this white line.

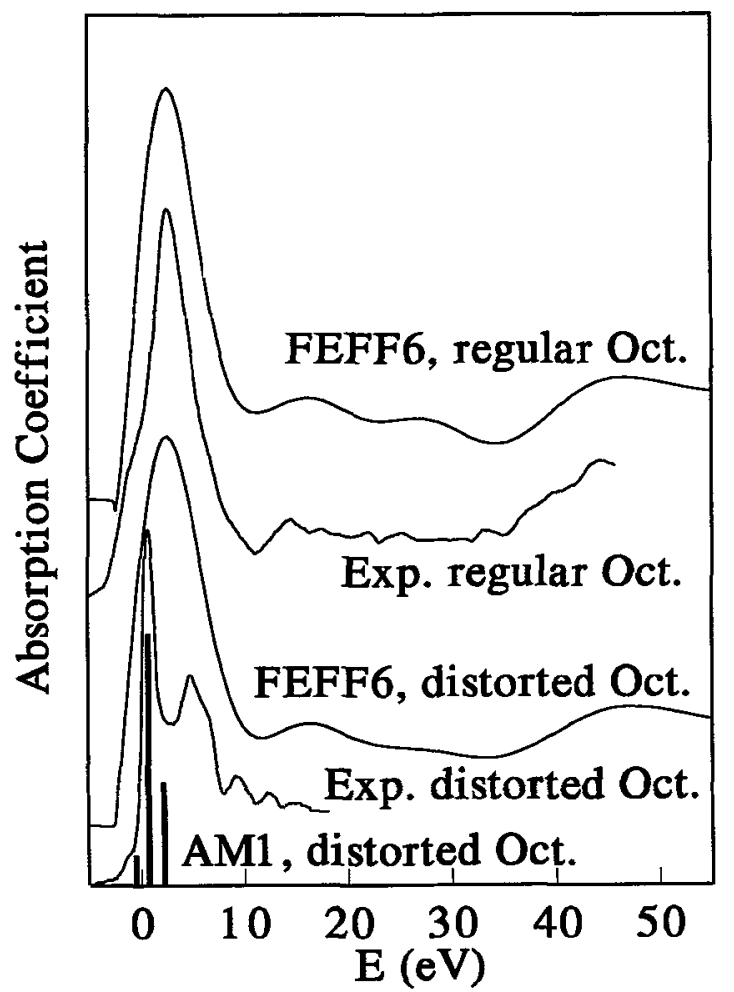

Figure 1: Experimental and theoretical (FEFF6 and AM1) $\mathrm{Al} \mathrm{K}$-edge NEXAFS data for distorted and nondistorted octahedral aluminum oxide compounds. The bar has arbitrary intensity and has been aligned with the "whiteline" peaks in the spectra. The bar represents an excitation into an unoccupied $\pi$-orbital.

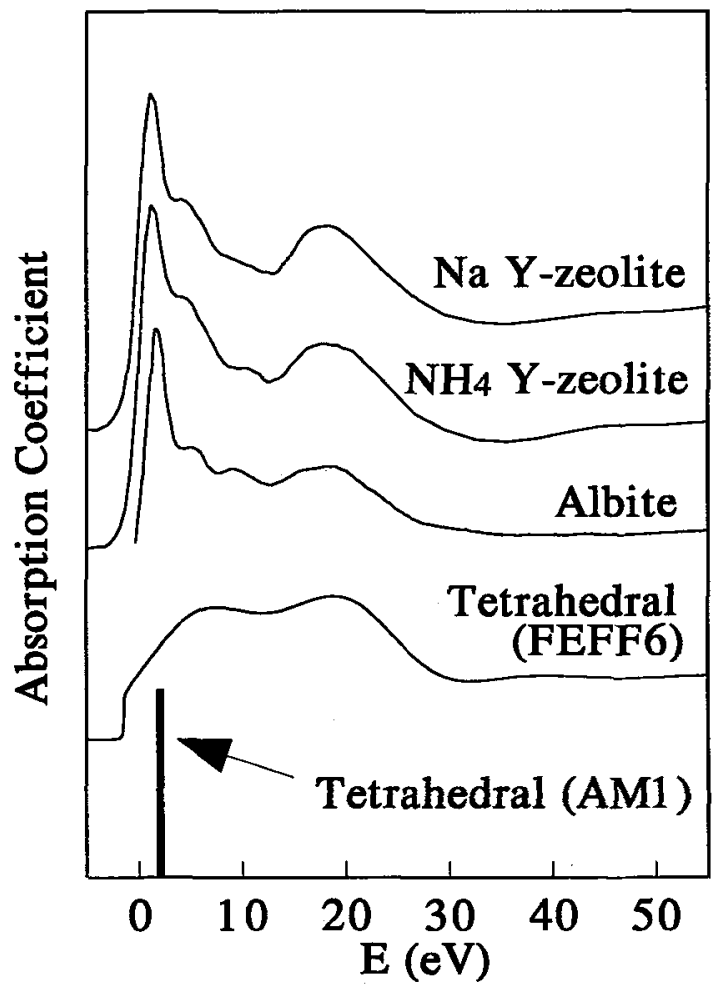

Figure 2: Experimental and theoretical (FEFF6 and AM1) Al K-edge NEXAFS data for compounds having tetrahedrally coordinated $\mathrm{Al}$ sites.

In addition to the shift of $2 \mathrm{eV}$ in white line position, the white line intensity of octahedrally coordinated aluminum oxide is higher than tetrahedrally coordinated aluminum oxide. This phenomenon of higher whiteline intensity for octahedrally coordinated aluminum sites can be checked by comparing the ratio of the whiteline intensity to the intensity well above 
threshold for several octahedral and tetrahedral compounds. All the comparable cases in the literature reveal a higher ratio for the octahedral compounds. An illustration of this can be found by comparing crystalline (octahedrally coordinated) and amorphous (mixed tetrahedrally / octahedrally coordinated) $\mathrm{Al}_{2} \mathrm{O}_{3}$, the first showing a higher white line ${ }^{26}$. It can be concluded that the NEXAFS spectra of octahedrally coordinated compounds have a higher whiteline intensity than spectra of tetrahedrally coordinated compounds.

Finally, tetrahedrally and octahedrally coordinated aluminum can be distinguished by the characteristic positions of peaks in the spectra. Moreover, distorted octahedral sites show a doublet whiteline in NEXAFS spectra.

\subsection{Y Zeolite AI NEXAFS and separation of different contributions}

In Figure 3 the $\mathrm{Al} \mathrm{K}$ edge NEXAFS spectra for $\mathrm{Y}$ zeolite with respectively $\mathrm{H}^{+}, \mathrm{NH}_{4}^{+}$and $\mathrm{Na}^{+}$as cations are given. Previous, EXAFS analysis indicates only one $\mathrm{Al}-\mathrm{O}$ bond length exists in each sample within the limits of accuracy ${ }^{13}$. $\mathrm{As}^{\mathrm{Al}}{ }^{27}$ NMR data indicated the presence of $15 \%$ octahedrally coordinated aluminum in the $\mathrm{H}^{+} \mathrm{Y}$ zeolite, this spectrum is renormalized on a tetrahedral atom basis. This was done by multiplying the spectrum with a factor 100/85 and then taking the difference with the spectra having only tetrahedral coordinated aluminum:

$$
\begin{aligned}
& \text { dif. }\left(\mathrm{Na}^{+} \mathrm{Y}\right)=100 / 85 * \operatorname{spectrum}\left(\mathrm{H}^{+} \mathrm{Y}\right)-\operatorname{spectrum}\left(\mathrm{Na}^{+} \mathrm{Y}\right) \\
& \text { dif. }\left(\mathrm{NH}_{4}^{+} \mathrm{Y}\right)=100 / 85 * \operatorname{spectrum}\left(\mathrm{H}^{+} \mathrm{Y}\right)-\operatorname{spectrum}\left(\mathrm{NH}_{4}^{+} \mathrm{Y}\right)
\end{aligned}
$$

The dif. $\left(\mathrm{Na}^{+} \mathrm{Y}\right)$ and dif. $\left(\mathrm{NH}_{4}^{+} \mathrm{Y}\right)$ spectra which represent that part of the original $\mathrm{H}^{+} \mathrm{Y}$ spectrum which is not due to tetrahedrally coordinated aluminium are given in Figure 3. In order to evaluate the influence of the multiplication factor on the difference spectra, to rule out accidental appearing characteristic features in the difference spectra, and to compensate for small errors in the NMR analysis, we used different multiplication factors, ranging from 100/75 to 100/95. In addition we checked the influence of the normalization which had been performed on the raw $Y$ zeolite spectra. Doing this, we artificially varied the normalization done on the raw data. The results (not shown) were spectra with basically similar shapes, only the relative amplitude of the peaks showed minor changes.

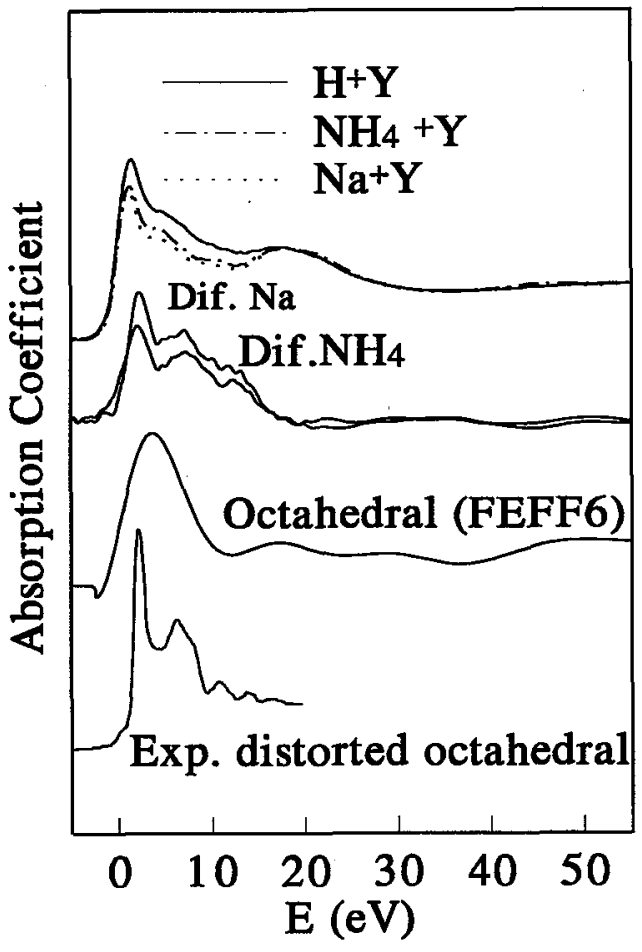

Figure 3: Comparison of Al K-edge NEXAFS data for $\mathrm{H}^{+} \mathrm{Y}$, $\mathrm{NH}_{4}+\mathrm{Y}$, and $\mathrm{Na}^{+} \mathrm{Y}$ zeolite materials. The difference (dif) spectra represent $\mathrm{H}^{+} \mathrm{Y}$ minus $\mathrm{Na}^{+} \mathrm{Y}$ and $\mathrm{H}^{+} \mathrm{Y}$ minus $\mathrm{NH}_{4}^{+} \mathrm{Y}$ respectively. For comparison, FEFF6 results for an octahedral cluster and an experimental distorted octahedral spectrum (Corundum) are shown.

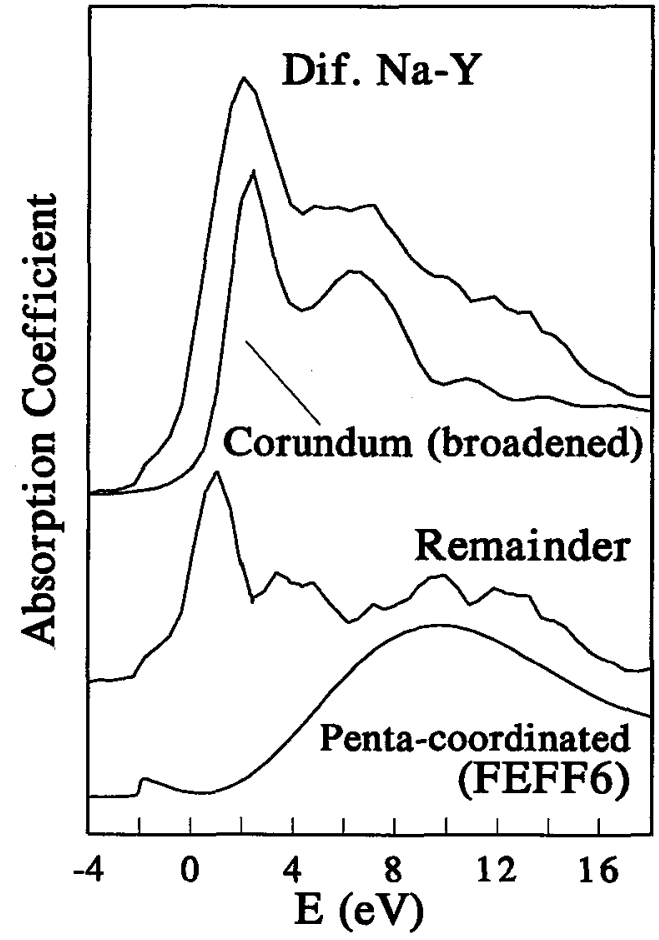

Figure 4: Comparison of the dif. Na-Y spectrum with corundum and the remainder between these two compared to FEFF6 results for penta-coordinated $\mathrm{AlO}_{5}$ cluster. 
In Figure 3 we see the whiteline peak position in the difference spectra is shifted to higher energy in comparison with the whiteline peak position in the $\mathrm{Na}^{+} \mathrm{Y}$ zeolite spectrum. Above we recognized this as a characteristic difference between spectra of octahedrally and tetrahedrally coordinated compounds. An experimental spectrum of a compound with aluminum in a distorted octahedral conformation (Corundum, Figure 1) is therefore shown. For comparison, a FEFF6 calculation on a $\mathrm{AlO}_{6}$ cluster with the aluminum octahedrally coordinated and having an aluminum oxide bond length of $1.85 \AA$ is added. The whiteline in the difference spectra is split by about $4 \mathrm{eV}$, equal to the splitting in the spectra of compounds in which the aluminum oxide octahedral is distorted. In the difference spectra a peak around $50 \mathrm{eV}$ can be distinguished and coincides with a peak in the FEFF6 calculated spectrum. In addition, it can be seen that the whiteline has a large intensity compared to the intensity at high energy ( $50 \mathrm{eV}$ above threshold). All these features, including the shift in whiteline, indicate that the difference spectrum can be attributed to distorted octahedrally coordinated aluminum oxide in the $\mathrm{H}^{+} \mathrm{Y}$ zeolite. This distorted octahedral has an aluminum oxide bond length of about $1.85 \AA$ indicated by the position of the peak at about $50 \mathrm{eV}$.

Although the similarities are evident, there is a distinct difference visible in the difference spectra and the corundum spectra, which can not be explained either by the presence of a cation, by differences in bond length distances as revealed by FEFF6 calculations (not shown), nor by the presence of the octahedrally coordinated aluminum oxide. This difference is a surplus of intensity in the difference spectra around 10 to $15 \mathrm{eV}$ above threshold compared to the spectrum for a distorted octahedrally coordinated compound.

Figure 4 compares the dif. NaY spectrum with that for Corundum on a smaller energy scale. The excess intensity around $7-15 \mathrm{eV}$ is clearly illustrated by taking the difference between these two spectra after broadening the experimental corumdum spectrum, where their relative normalization is optimally chosen so that the intensity of the remainder has reasonable magnitude at higher energy. This remainder spectrum now also reveals excess intensity around 2-3 eV. Note also that these excess intensity peaks do not align well with peaks in the spectra found for $\mathrm{Na}^{+} \mathrm{Y}$, where the Al sites are all tetrahedrally coordinated.

As already mentioned in the introduction, conventional NMR does not indicate the presence of any non framework penta coordinated aluminum or distorted tetrahedrally distorted aluminum oxide. But $\mathrm{Al}^{27}$ MAS NMR indicates that the presence of octahedrally coordinated aluminum oxide coincides with the (possible) presence of some penta-coordinated aluminum oxide, or highly distorted tetrahedral $\mathrm{Al}$ sites. We therefore performed FEFF6 calculations on two clusters $\left(\mathrm{AlO}_{5}\right)$ in which the aluminum is respectively square pyramidal and trigonal bipyramidal coordinated. The aluminum oxygen bond length for these calculations is $1.65 \AA$. In addition we performed calculations on identical clusters, except for an enlarged aluminum oxide bond length $(1.70 \AA$ and $1.75 \AA)$. The result is a slight shift of the whiteline to lower energy, but in any case these calculated NEXAFS spectra show a large intensity in the range 10-15 eV above threshold. This is clearly demonstrated by the spectra presented in Figure 4 in which a calculated penta-coordinated spectrum is compared to the remainder spectrum. The excess intensity around $2-3 \mathrm{eV}$ probably corresponds to an excitonic peak in the spectra for penta-coordinated aluminum, which the FEFF6 code does not reproduce, as we found previously for the tetrahedrally coordinated aluminum.

It can not be concluded that any distorted tetrahedrally coordinated aluminum oxide is present in the $\mathrm{H}^{+} \mathrm{Y}$ zeolite on basis of the NEXAFS result. FEFF6 calculations on $\mathrm{AlO}_{4}$ clusters in distorted tetrahedral conformation (not shown) show a large decrease in intensity from $5 \mathrm{eV}$ to about $30 \mathrm{eV}$. Figure 4 does not show any evidence for the presence of this distorted tetrahedrally coordinated aluminum oxide.

These NEXAFS results strongly suggest the presence of penta-coordinated aluminum oxide in the $\mathrm{H}^{+} \mathrm{Y}$ zeolite, accompanying the distorted octahedrally (non-framework) and the regular tetrahedrally coordinated (framework) aluminum oxide. The Al-O bond length distances are circa $1.70 \AA$ (penta coordinated), $1.85 \AA-1.90 \AA$ (octahedrally coordinated) and circa $1.70 \AA$ (tetrahedrally coordinated) respectively.

\section{CONCLUSIONS}

The Al K edge NEXAFS spectra of various minerals and Y zeolites are successfully interpreted starting at around 5 $\mathrm{eV}$ above threshold using the curved-wave multiple scattering cluster calculations developed in FEFF6. Octahedrally and tetrahedrally coordinated aluminum could be distinguished. Characteristic features for the different conformations are identified. A semi-empirical molecular orbital calculation is used to interpret the NEXAFS below $5 \mathrm{eV}$ above threshold. A distorted octahedral conformation could be distinguished from a regular octahedral conformation. For the distorted octahedral, a new assignment is given to the transitions of the whiteline.

Using the established characteristic features in the NEXAFS lineshapes for each coordination geometry, separate contributions to the NEXAFS lineshape of $\mathrm{H}^{+} \mathrm{Y}$ zeolite could be identified. In addition to the tetrahedral conformation the presence of distorted octahedrally coordinated aluminum could be assigned. Finally, there are strong indications for the presence of penta-coordinated aluminum in the $\mathrm{H}^{+} \mathrm{Y}$ zeolite accompanying the extra-framework octahedrally coordinated aluminum. However, at present the experimental lineshape does not allow a determination of the exact geometry of the pentacoordinated aluminum sites.

Interpretation of NEXAFS data has proven to be a successful tool in providing unique geometrical information on the Al coordination. In the future, additional work will be done to improve the separation of the different contributions to the NEXAFS data for the Y zeolites. These data will be compared to NMR and the correlation with the TOF will be investigated. 


\section{Acknowledgements}

This work was supported by the Office of Naval Research (USA) and by the Catalysis Center Utrecht (NL). The authors thank H. Qian and T. Nabi (George Washington University) for their interesting and helpful discussions.

\section{References}

1 Van Santen, R.A., Kramer, G.J., Chem. Rev. 95, (1995), 637.

2 Farneth, W.E., Gorte, R.J., Chem. Rev. 95, (1995), 615.

3 Fyfe, C.A., Mueller, K.T., Kokotailo, G.T. in NMR Techniques in Catalysis, eds. Bell, A.T. and Pines, A. (Marcel Dekker, Inc, NY) 1994.

4 Samoson, A, Lippmaa, E., Phys. Rev. B, 28, (1983) 6567.

5 Samoson, A., Lippmaa, E., Engelhardt, G., Lohse, U., Jerschkewitz, G., Chem. Phys. Letters 134, (1987) 589.

6 Freude, D., Brunner, E., Pfeifer, H., Prager, D., Jerschkewitz, H.-G., Lohse, U., and Oelmann, G., Chem. Phys. Letters 139 , (1987) 325.

7 Chen, F.R., Davis, J.G., and Fripiat, J.J., J. Catal. 133, (1992) 263.

8 Gilson, J.-P., Edwards, G.C., Peters, A.W., Rajagopalan, K., Wormsbecher, R.F., Roberie, T.G., and Satlock, M.P., J. Chem Soc. Chem Comm. 91, (1987).

9 Sanz, J., Fornés, V. and Corma, A., J. Chem. Soc. Faraday Trans I, 84,(9), (1988) 3113.

10 Grobet, P.J., Geerts, H., Martens, J.A. and Jacobs, P.A., J. Chem. Soc., Cem. Comm., (1987) 1688.

11 Pellet, R.J., Blackwell, C.S., and Rabo, J.A., J. Catal. 114, (1988) 71 and references therein.

12 Lonyi, F, Lunsford, J.H., J.Catal., 136, (1992) 566.

13 Koningsberger, D.C., and Miller, J.T. in Studies in Surface Science and Catalysis 101B, eds. Hightower, J.W., Delgass, W.N., Iglesia, E., Bell, A.T., ElsevierAmsterdam, (1996) 841.

14 Sohn, J.R., Decanio, S.J., Fritz, P.O., Lunsford, J.H., J. Phys. Chem. 90, (1986) 4847.

15 "Hyperchem" Release 3 for Windows Autodesk, Inc., Sausalito, CA, (1993).

16 Mustre de Leon, J., Rehr, J. J., Zabinsky, S.I. and Albers, R.C., Phys. Rev. B 44, (1991) 4146.

17 Rehr, J.J., , J. Appl. Phys. Supplement, 32, 1993) 8.

18 Schwarz, W.H.E., Chem. Phys. 13, (1976) 363.

${ }^{19}$ Landron, C., Badets, M.C., Douy, A., Coutures, J., Coutures, J.P., Daniel, P., and Flank, A.M., Phys. Stat. Sol.(b), 167, (1991) 429.

20 Wyckoff, R.W.G., Crystal Structure, Vol. 1 second edition, John Wiley and Sons, New York (1963).

21 Waychunas, G.A., Brown Jr., G.E. in EXAFS and Near Edge Structures III, eds. Hodgts, K.A., Hedman, B. and Penner-Hahn, J.E., Springer-Verlag, Berlin (1984) 336

22 McKeown, D.A., Waychuna, G.A., Brown Jr., G.E., J. Non-Cryst. Solids 74 , (1985) 349.

23 Tossell, J.A., J. Phys. Chem. Solids 36 , (1975) 1273.

24 Li,D., Bancroft, G.M., Fleet, M.E., Feng, X.H., Pan, Y., American Mineralogist, 80, (1995) 432.

25 Not published data.

26 Kamijo, N., Kageyama, Kacabata, K., Nishihagi, K.,Uehara, Y., and Taniguchi, K., in X-ray Absorption Fine Structure, ed. Sahar, S., Ellis Horwood, New York, (1991) 613. 